\title{
Oral gastrografin radiography for the evaluation of the functional impact of peritoneal carcinomatosis: Correlation with clinicopathological findings
}

\author{
LIE-JUN MEI ${ }^{1,2^{*}}$, LIN-WEI WANG ${ }^{1,3^{*}}$, CHAO-QUN HUANG ${ }^{1,3}$, XIAO-JUN YANG ${ }^{1,3}$ and YAN LI ${ }^{1,3}$ \\ Departments of ${ }^{1}$ Oncology and ${ }^{2}$ Radiology, Zhongnan Hospital of Wuhan University; ${ }^{3}$ Hubei Key Laboratory \\ of Tumor Biological Behaviors, Hubei Cancer Clinical Study Center, Wuhan, Hubei 430071, P.R. China
}

Received January 29, 2015; Accepted April 15, 2015

DOI: $10.3892 / \mathrm{mco} .2015 .573$

\begin{abstract}
This study was conducted to evaluate the functional impact of peritoneal carcinomatosis (PC) on the gastrointestinal system by oral gastrografin radiography (OGR). OGR was performed on 105 patients with PC from abdominal malignancies. The OGR characteristics were analyzed and compared with intraoperative observations. OGR provided real-time dynamic information on the functional impacts of PC. The OGR findings were normal in $9(8.6 \%)$ and abnormal in $96(91.4 \%)$ cases. In terms of frequency, 33 cases (31.4\%) exhibited mild intestinal aggregation and flattening of the intestinal mucosa; 29 cases (27.6\%) exhibited limited intestinal invasion, marginally stenotic small bowel and mucosal deformities; 26 cases (24.8\%) exhibited only mild mesenteric contracture and mild slowing of gastrointestinal peristalsis; 5 cases (4.8\%) exhibited obvious mesenteric contracture, ball-like changes, fixed position and disappearance of the intestinal mucosa; 2 cases $(1.9 \%)$ exhibited complete pyloric obstruction; and 1 case (0.9\%) exhibited duodenal obstruction. Gastric PC was associated with a higher percentage of stomach filling defects and small intestinal aggregates compared with PC from other malignancies ( $\mathrm{P}<0.01$ for both). In 87 cases, the ORG findings were in accordance with the intraoperative findings $(\kappa=0.726, \mathrm{P}<0.001)$, whereas 17 cases $(16.2 \%)$ were underestimated and $1(0.9 \%)$ was overestimated by OGR. This study indicated that OGR may be a useful technique for the evaluation of the functional impacts of $\mathrm{PC}$ on the gastrointestinal system and may help optimize the selection of patients for treatment.
\end{abstract}

Correspondence to: Professor Yan Li, Department of Oncology, Zhongnan Hospital of Wuhan University, 169 Donghu Road, Wuchang, Wuhan, Hubei 430071, P.R. China

E-mail: liyansd2@163.com

*Contributed equally

Key words: peritoneal carcinomatosis, oral gastrografin radiography, cytoreductive surgery, hyperthermic intraperitoneal chemotherapy

\section{Introduction}

Peritoneal carcinomatosis (PC) is a common form of metastatic dissemination into the peritoneal cavity, frequently from cancers of the stomach, colon, ovary or pancreas $(1,2)$. Our previous studies demonstrated that the survival of PC patients has significantly improved since the introduction of cytoreductive surgery (CRS) plus hyperthermic intraperitoneal chemotherapy (HIPEC) and modern systemic therapies (3-6). To ensure that patients optimally benefit from this multimodality treatment, patient selection must be restricted to those with limited peritoneal disease, in whom a complete CRS may be achieved $(7,8)$.

Modern medical imaging technologies play an important role in this regard. Contrast-enhanced multidetector-row computed tomography (CT) is the modality of choice in the preoperative evaluation of PC patients (9-13). However, although modern CT technology has significantly contributed to the determination of the PC score, it is a relatively static technology, unable to accurately observe the bowel movement and dynamic distribution of the small intestines. Therefore, there is an urgent clinical demand for dynamic imaging technologies for real-time observation of the changes of the small intestine, in order to accurately assess the severity of the impact of $\mathrm{PC}$ on bowel function.

There are currently several techniques for the observation of the bowel movement under X-ray screen, such as barium sulfate swallowing and air-barium sulfate double-contrast studies. However, these studies are not suitable for PC patients, as these patients usually have intestinal obstruction and barium sulfate swallow may exacerbate the clinical symptoms of PC. Therefore, a suitable alternative is required. For this purpose, water-soluble contrast media are promising options.

This study aimed to prospectively investigate the clinical utility of oral gastrografin radiography (OGR) for the real-time dynamic study of the functional impacts of PC on the gastrointestinal system, to enable the selection of patients suitable for CRS+HIPEC treatment.

\section{Patients and methods}

Patients. A total of 105 patients with PC were included in this study, including 54 cases (51.5\%) with PC from gastric cancer, 
22 (21.0\%) from colorectal cancer, 9 (8.7\%) from ovarian cancer, 6 (5.7\%) from primary peritoneal carcinoma, $6(5.7 \%)$ from appendiceal mucinous adenocarcinoma, 2 (1.9\%) from gallbladder carcinoma, 2 (1.9\%) from lung cancer, 1 (0.9\%) from breast cancer, $1(0.9 \%)$ from splenic adenocarcinoma, $1(0.9 \%)$ from malignant mesothelioma and $1(0.9 \%)$ from squamous cell carcinoma of the uterine cervix. The patients included 43 men $(41.0 \%)$ and 62 women $(59.0 \%)$, with an age range of 22-76 years (median, 55 years).

The study protocol was approved by the Institutional Review Board of the Zhongnan Hospital of Wuhan University (Wuhan, China) and conformed to the ethical standards of the World Medical Association Declaration of Helsinki. Written informed consent was obtained from the patients prior to conducting this examination.

\section{OGR study}

Preparation. In patients with $\mathrm{PC}$ or suspected PC, the gastrointestinal motility is often slower than normal (14). Therefore, proper preparation is crucial, including $4 \mathrm{~h}$ of fasting and water deprivation prior to the exanimation, to ensure the upper digestive tract is empty. For patients with intestinal obstruction, gastrointestinal decompression must be performed prior to the examination, to reduce the retention of excessive gastrointestinal fluid.

OGR study at the standing position. After receiving detailed instructions on the entire examination procedure, each patient stood on the digital gastrointestinal machine for a brief observation of the chest and the abdomen. If considered suitable for such an examination, the patient was instructed to swallow $80 \mathrm{ml}$ of the hyperosmolar water-soluble contrast medium gastrografin $(76 \%$ compound meglumine diatrizoate injection, $20 \mathrm{ml} / \mathrm{ampule}$, containing $15.2 \mathrm{~g}$ meglumine diatrizoate and sodium diatrizoate; Shanghai Xudong Haipu Pharmaceutical Co., Ltd., Shanghai, China), and directly observed under the Philips OMEN type digital gastrointestinal machine (OmniDiagnost Eleva Release DI 1.1; Philips, Amsterdam, The Netherlands). The flow trace of gastrografin was recorded (15).

OGR study at shifting positions. In patients fulfilling the required conditions, when the contrast medium was observed to have reached the stomach, the patient was placed in the supine position. The outline and motility of the stomach and duodenum were first observed while the patient changed positions on the examination bed. Once the contrast medium was observed to have reached the proximal jejunum, the patient shifted to the standing position and the flow trace of the contrast agent was recorded at regular time intervals (15 min) to reflect the movements and distributions of the small intestine, until the contrast agent reached the cecum.

Key considerations of the study. As the aim of this study was to evaluate the functional impacts of PC on the gastrointestinal system, attention was focused on the following aspects during the study: i) Stomach filling and movements: Once the contrast agent reached the stomach, the contour and movement of the stomach were observed. Any disruptions of the mucosa, gastric wall recesses and/or irregular movements were recorded. The time interval from the entry of the contrast agent to the emptying of the stomach was also recorded. ii) Small intestinal filling, movements and distribution: When the contrast agent reached the duodenum, the contour, distribution, mucosal characteristics and movements of the different parts of the small intestine were observed, recording the following information: Stenosis of the small bowel, stiffness of the intestinal wall, deformation or mucosal damage, abnormal distribution of peristalsis, site and severity of intestinal obstruction. iii) Contracture of the small intestinal mesentery: As PC frequently involves the small intestinal mesentery, leading to contracture and hardening, a number of typical abnormal signs may appear during the examination. In patients with partial mesenteric contracture, the typical sign is the formation of small intestinal aggregates in one or several segments of the small intestine, with reduced motility of the involved part of the small intestine. In patients with more severe mesenteric contracture, ball-like or petal-like aggregates of small intestinal loops may appear, with significantly reduced flow of the contrast agent through them. In patients with generalized mesenteric contracture, the entire small intestine may be pulled together to form a fixed ball in the central abdomen, with stagnation of the contrast agent within the intestinal loops.

Comparison of OGR signs with clinicopathological findings. The patients underwent CRS+HIPEC, with the designated operating team and standardized protocols established at our institution (6). Briefly, following induction of general anesthesia, a midline xiphoid-to-pubic incision was performed and maximal CRS was performed, with removal of the primary tumor with acceptable margins, any involved adjacent tissues/organs and regional lymph nodes, and peritonectomy. The completeness of cytoreduction was evaluated prior to HIPEC (16). The intraoperative findings were recorded and compared with the OGR findings.

Statistical analysis. Data analyses were performed using the SPSS software, version 17.0 (SPSS Inc., Chicago, IL, USA). The numerical data were directly recorded and the categorical data were recorded into different categories. The OGR characteristics of PC from different cancers were compared and tested using analysis of variance (Chi-squared test). The accordance rate between OGR and operative findings was assessed with correlation studies. A two-sided P-value of $<0.05$ was considered to indicate statistically significant differences.

\section{Results}

Clinicopathological characteristics of PC patients. A total of 105 patients were included in this study. The major clinicopathological characteristics are summarized in Table I.

Technical considerations of $O G R$. All the patients completed the OGR (103 patients by direct oral swallow and 2 patients by nasogastric tube infusion). The time interval between the administration of gastrografin to the completion of the examination ranged from 30 to $360 \mathrm{~min}$ (median, $90 \mathrm{~min}$; mean $\pm \mathrm{SD}, 103 \pm 9.1 \mathrm{~min})$. In 98 patients the contrast agent successfully reached the cecum, while in 7 patients the contrast agent failed to reach the cecum due to total pyloric obstruction $(n=2)$, total intestinal obstruction $(n=3)$ and loss of intestinal mobility $(\mathrm{n}=2)$. 
Table I. Major clinicopathological characteristics of the patients in this study $(\mathrm{n}=105)$.

\begin{tabular}{lc}
\hline Variables & Patient no. $(\%)$ \\
\hline Median age, years (range) & $55(22-76)$ \\
Gender, male/female & $43 / 62(41.0 / 59.0)$ \\
Primary tumor & \\
Gastric cancer & $54(51.4)$ \\
Colorectal cancer & $28(26.7)$ \\
Gynecological cancer & $9(8.6)$ \\
Primary peritoneal carcinoma & $6(5.7)$ \\
Others & \\
Histopathology & $8(7.6)$ \\
Adenocarcinoma & \\
Well/moderately differentiated & \\
Poorly differentiated/undifferentiated & $36(34.3)$ \\
Signet ring cell carcinoma & $11(10.5)$ \\
Mucinous adenocarcinoma & $29(27.6)$ \\
Serous papillary adenocarcinoma & $4(3.8)$ \\
Others & $8(7.6)$ \\
Degree of small intestinal & \\
mesenteric contracture & \\
Normal & \\
Mild & $39(37.1)$ \\
Intermediate & $51(48.6)$ \\
Severe & $5(4.8)$ \\
\hline
\end{tabular}

${ }^{a}$ Including peritoneal metastasis from breast cancer $(\mathrm{n}=1)$, lung cancer $(n=2)$, gallbladder cancer $(n=2)$, splenic adenocarcinoma $(n=1)$, malignant peritoneal mesothelioma $(n=1)$ and squamous cell carcinoma of the uterine cervix $(n=1)$.

Normal OGR findings in PC patients without small intestinal function impairment. Of the 105 patients, 9 (8.6\%) exhibited normal OGR findings. The anatomical position, outlines, distribution and motility of the stomach and small intestine were normal, with the mucosa of the jejunum exhibiting feather-like or snowflake-like characteristics and the mucosa of the ileum exhibiting strip-like characteristics. The intestinal peristalsis was normal, with gastrografin passing through the small intestine to the cecum in 60-90 min (Fig. 1).

Abnormal OGR findings in PC patients with small intestinal function impairment. Of the 105 patients, 96 (91.4\%) exhibited abnormal OGR findings. A total of 29 cases (27.6\%) exhibited limited intestinal invasion, marginally stenotic small bowel and mucosal deformities; 5 (4.8\%) exhibited obvious mesenteric contracture, ball-like changes, fixed position and disappearance of the intestinal mucosa; 33 (31.4\%) exhibited normal intestinal peristalsis, mild intestinal aggregation and flattening of the intestinal mucosa; 26 (24.8\%) exhibited only mild mesenteric contracture and mild slowing of the gastrointestinal peristalsis; 2 (1.9\%) exhibited total pyloric obstruction and $1(0.9 \%)$ exhibited duodenal obstruction, with no contrast medium in the small intestine.

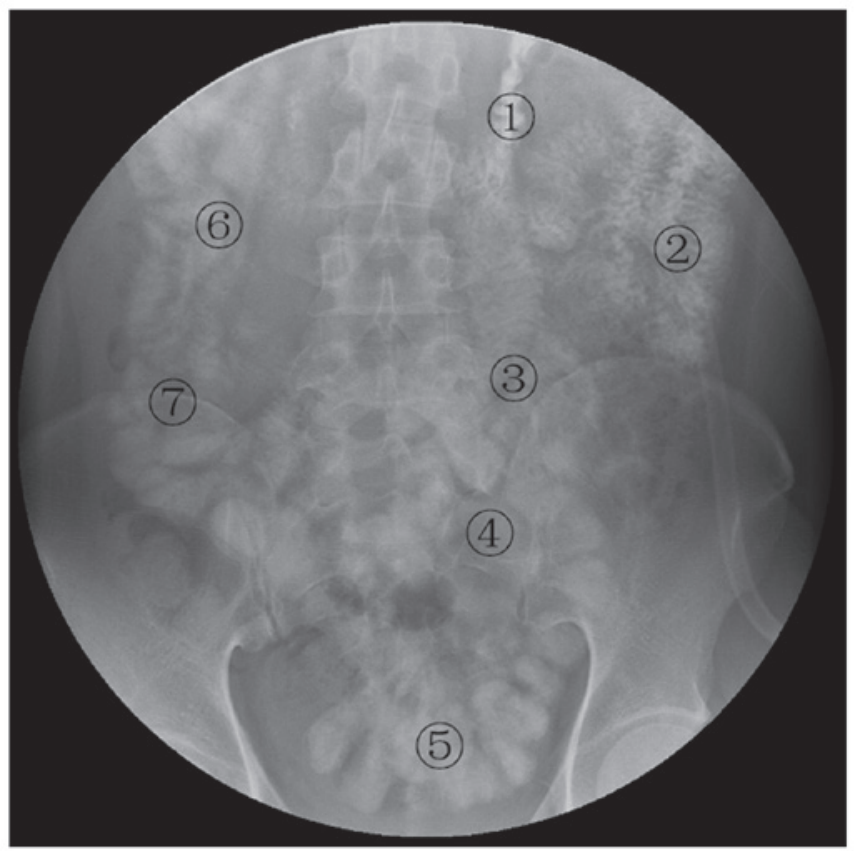

Figure 1. OGR characteristics of the small intestine in normal subjects. The jejunum exhibits a normal lumen without expansion; the contrast medium passed through the first part of the jejunum in $\sim 5 \mathrm{~min}(1)$, the second part of the jejunum in 10-15 min (2) and the third part of the jejunum in 15-20 min (3). At the jejunum-ileum transitional region (4) the intestinal lumen exhibited no expansion and clear feather-like or strip-like mucosa; the contrast medium passed through this region in $\sim 15 \mathrm{~min}$. The proximal ileum exhibited clear strip-like mucosa and the contrast medium passed through in $\sim 20 \mathrm{~min}$ (5). At the distal ileum, the intestinal lumen exhibited no expansion, with clear strip-like mucosa; the contrast medium passed through in $\sim 15 \mathrm{~min}(6)$. The ileocecal junction and colon exhibited no expansion; the ileocecal valve and haustrum of the colon were clear and the contrast medium passed through the ileocecal junction in $2-5 \mathrm{~min}(7)$.

In patients with limited intestinal invasion, the involved small bowel loop became stenotic, with disordered mucosa exhibiting serrated changes (Fig. 2A1, white arrows) and segmental intestinal dilation due to incomplete obstruction (Fig. 2A2, black arrows); however, the distribution of the bowels remained normal. In cases with more severe small intestinal invasion, incomplete intestinal obstruction with proximal bowel dilation was observed (Fig. 2B2, black arrows).

In patients with mild mesenteric contracture, the small bowel loops became slightly aggregated and relatively fixed (Fig. 2C1, white arrow), although the distribution appeared normal. The wall of the involved intestines appeared mildly stiff, with a typical stiff ' 3 ' sign (Fig. 2C2, black arrows), although the small bowel exhibited no stenosis and the intestinal mucosa was relatively flat. The dynamic radiography revealed slow intestinal peristalsis, with the contrast medium requiring $>120 \mathrm{~min}$ to reach the ileocecum.

In cases with intermediate mesenteric contracture, the involved small intestines exhibited cluster buckling and aggregation (Fig. 2D1, black ellipse). The intestinal distribution appeared normal, without expansion or stenosis. On dynamic radiographic observation, there was slowing of the peristalsis of the involved intestine, whereas the uninvolved intestinal loops appeared normal (Fig. 2D2).

In cases with mixed mesenteric contracture and limited intestinal invasion, the involved small intestinal loops became 

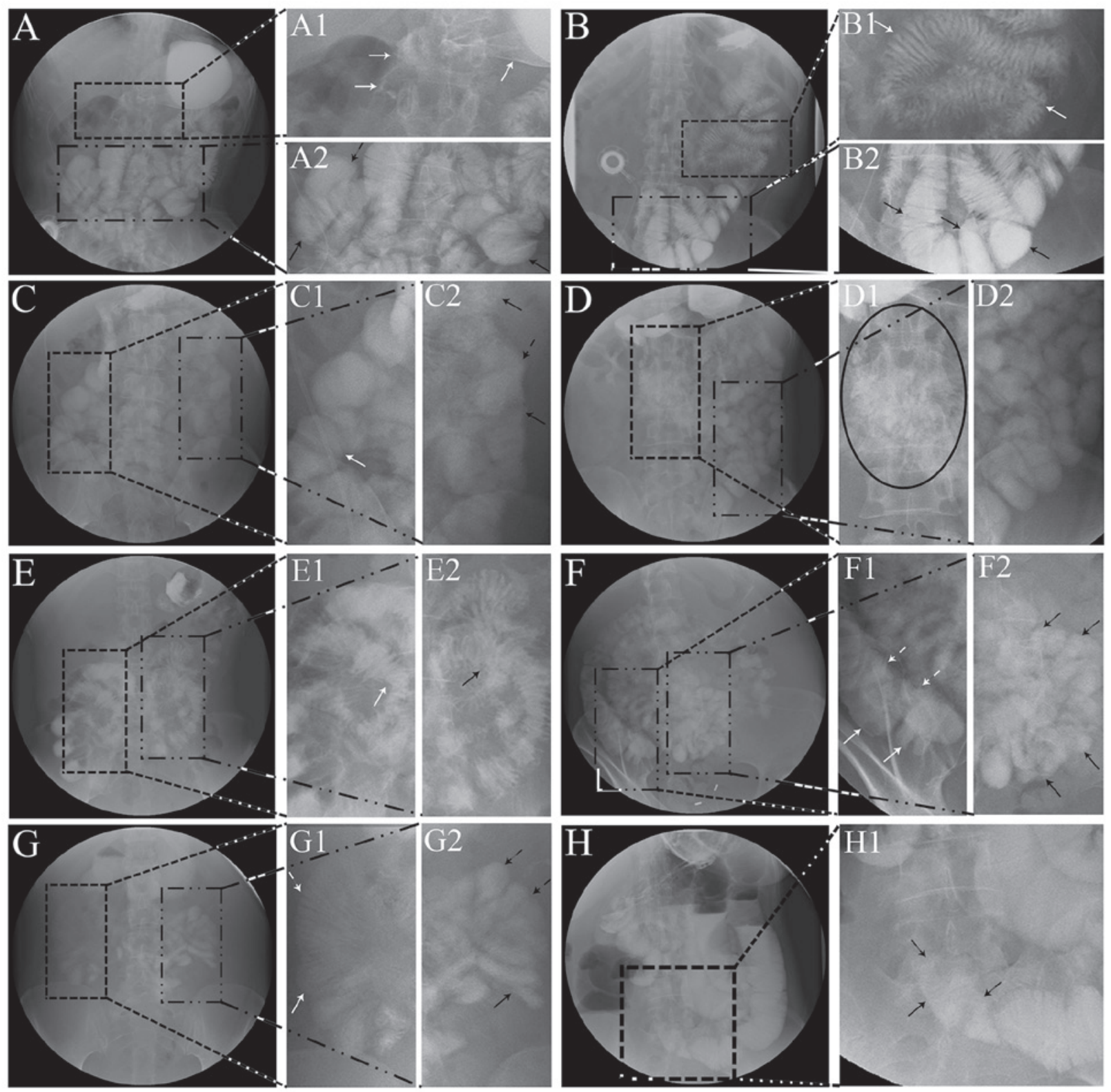

Figure 2. Oral gastrograffin radiography findings in peritoneal carcinomatosis. (A) Limited intestinal invasion, with signs of serrated changes of the mucosa in the proximal jejunum (A1, white arrows) and segmental intestinal dilation due to incomplete obstruction (A2, black arrows). (B) More severe distal jejunum invasion, with dilation of the proximal jejunum (B2, black arrows); the distribution and peristalsis of the proximal jejunum were normal (B1, white arrows). (C) Intestinal distribution, mild bowel aggregation and relatively fixed position of the distal ileum at the right lower quadrant (C1, white arrow); proximal jejunum at the left upper quadrant exhibiting a typical stiff ' 3 ' sign (C2, black arrows). (D) Intermediate mesenteric contracture exhibiting the 'cluster sign' (D1, black ellipse); the peristalsis and distribution were normal in the remaining intestine (D2). (E) Asymmetric mucosa of the involved small bowel exhibiting a half moon-shaped pressure recesses sign (E1, white arrow); the involved small bowel exhibited a fixed position and fuzzy intestinal mucosa (E2, black arrow). (F) The ascending colon exhibited a distinct haustrum (F1, white arrows); ball-like sign of mesenteric contracture with unclear boundary (F2, black arrows). (G) Total obstruction of the distal intestine (G1, white arrows), with petal-like mesenteric contracture (G2, black arrows). (H) Significant stenosis of the terminal ileum and complete intestinal obstruction (H1, black arrows).

segmented and aggregated, leading to altered anatomical positions. On dynamic observation, part of the involved small intestine exhibited asymmetric stenosis, with a half moon-shaped pressure recess sign in the mucosa (Fig. 2E1, white arrow); the aggregated intestines exhibited fixed position and fuzzy intestinal mucosa (Fig. 2E2, black arrow).

In cases with severe mesenteric contracture, the involved small intestinal loops became distinctly fixed and aggregated, exhibiting ball-like or petal-like signs. The intestinal mucosa was ill-defined and the jejunum and ileum could not be easily defined. On dynamic observation, the intestinal peristalsis was very slow, with the contrast agent mainly moving due to gravity rather than through peristalsis. In certain cases with more severe mesenteric invasion, the aggregated small intestines exhibited a distinct ball-like change (Fig. 2F2, black arrows); other cases with severe mesenteric invasion exhibited petal-like aggregates (Fig. 2G2, black arrows), with total obstruction of the distal intestine (Fig. 2G1, white arrows).

In cases of severe intestinal invasion, severe stenosis or complete obstruction of the involved segment of the small intestine was observed and the proximal bowel became markedly expanded. On dynamic observation, there was enhanced 
Table II. Major abnormal findings on oral gastrografin radiography in the 105 patients with peritoneal carcinomatosis (PC).

\begin{tabular}{|c|c|c|c|c|c|}
\hline Abnormal findings, no. (\%) & $\begin{array}{c}\text { Gastric PC } \\
(\mathrm{n}=54)\end{array}$ & $\begin{array}{c}\text { Colorectal PC } \\
\quad(\mathrm{n}=28)\end{array}$ & $\begin{array}{c}\text { Gynecological PC } \\
(\mathrm{n}=9)\end{array}$ & $\begin{array}{c}\text { Other PC } \\
(n=14)\end{array}$ & P-value \\
\hline \multicolumn{6}{|l|}{ Stomach } \\
\hline Filling defect & $21(38.9)$ & $0(0.0)$ & $0(0.0)$ & $0(0.0)$ & 0.000 \\
\hline Gastric wall recess & $15(27.8)$ & $8(28.6)$ & $0(0.0)$ & $4(28.6)$ & 0.332 \\
\hline Reduced motility/stagnation & $38(70.4)$ & $16(57.1)$ & $3(33.3)$ & $10(71.4)$ & 0.138 \\
\hline \multicolumn{6}{|l|}{ Small intestine } \\
\hline Intestinal wall rigidity/spasm & $9(16.7)$ & $3(10.7)$ & $1(11.1)$ & $2(14.3)$ & 0.893 \\
\hline Mucosal disruption & $9(16.7)$ & $7(25.0)$ & $3(33.3)$ & $4(28.6)$ & 0.557 \\
\hline Luminal stenosis & $15(27.8)$ & $10(35.7)$ & $4(44.4)$ & $5(35.7)$ & 0.630 \\
\hline Reduced peristalsis/stagnation & $38(70.4)$ & $19(67.9)$ & $5(55.6)$ & $12(85.7)$ & 0.635 \\
\hline \multicolumn{6}{|l|}{ Mesenteric contracture } \\
\hline Small aggregates & $38(70.4)$ & $10(35.7)$ & $4(44.4)$ & $5(55.6)$ & 0.008 \\
\hline Ball-like, petal-like aggregates & $6(11.1)$ & $3(10.7)$ & $0(0.0)$ & $1(7.1)$ & 0.743 \\
\hline One large central ball & $4(7.4)$ & $0(0.0)$ & $1(11.1)$ & $0(0.0)$ & 0.292 \\
\hline
\end{tabular}

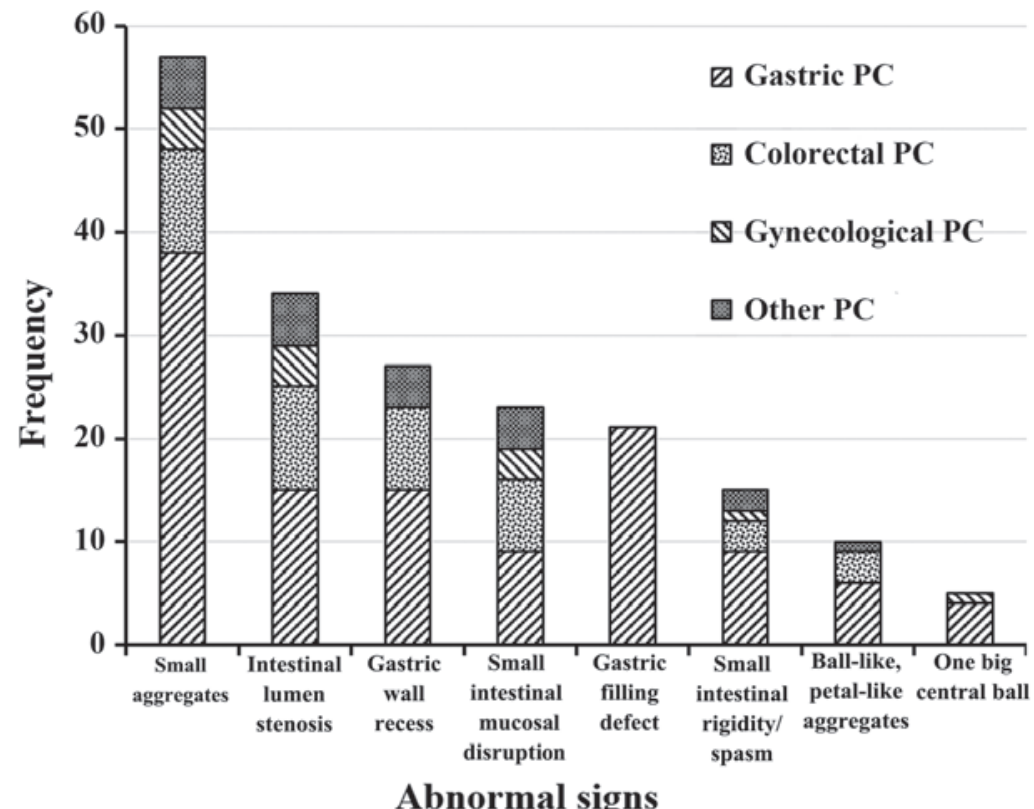

Figure 3. Distribution graph of 192 abnormal signs on oral gastrografin radiography in peritoneal carcinomatosis (PC).

reverse peristalsis of the proximal intestine. There were also marked intestinal cavity liquid gas planes (Fig. 2H).

Statistical analysis of abnormal OGR signs. Abnormal OGR signs were often composite, with a total of 192 abnormal signs observed in this group. The frequency of these OGR findings is presented in Fig. 3 and the detailed statistical analysis is presented in Table II.

Comparison between $O G R$ and clinicopathological findings. In the 96 cases with abnormal OGR findings, a total of 192 typical abnormal signs were recorded. These abnormal signs were further compared with the intraoperative findings. In 87 cases (82.9\%), the ORG findings were in accordance with the intraoperative findings $(\kappa=0.726, \mathrm{P}<0.001)$ (Fig. 4). Among the 18 cases with discordant findings, 17 (16.2\%) were underestimated by OGR and turned out to be more severe during surgery, whereas 1 case $(0.9 \%)$ was overestimated as severe mesenteric contracture on ORG and was found to be intermediate mesenteric contracture during surgery. Among the 17 underestimated cases, $9(8.6 \%)$ were upstaged from normal to mild mesenteric contracture, 2 (1.9\%) were upstaged from normal to intermediate mesenteric contracture, 2 (1.9\%) with mild intestinal expansion and limited intestinal wall invasion were upstaged from mild to intermediate mesenteric contracture, $2(1.9 \%)$ were upstaged from mild to severe mesenteric contracture and $2(1.9 \%)$ were upstaged from intermediate to severe mesenteric contracture. 

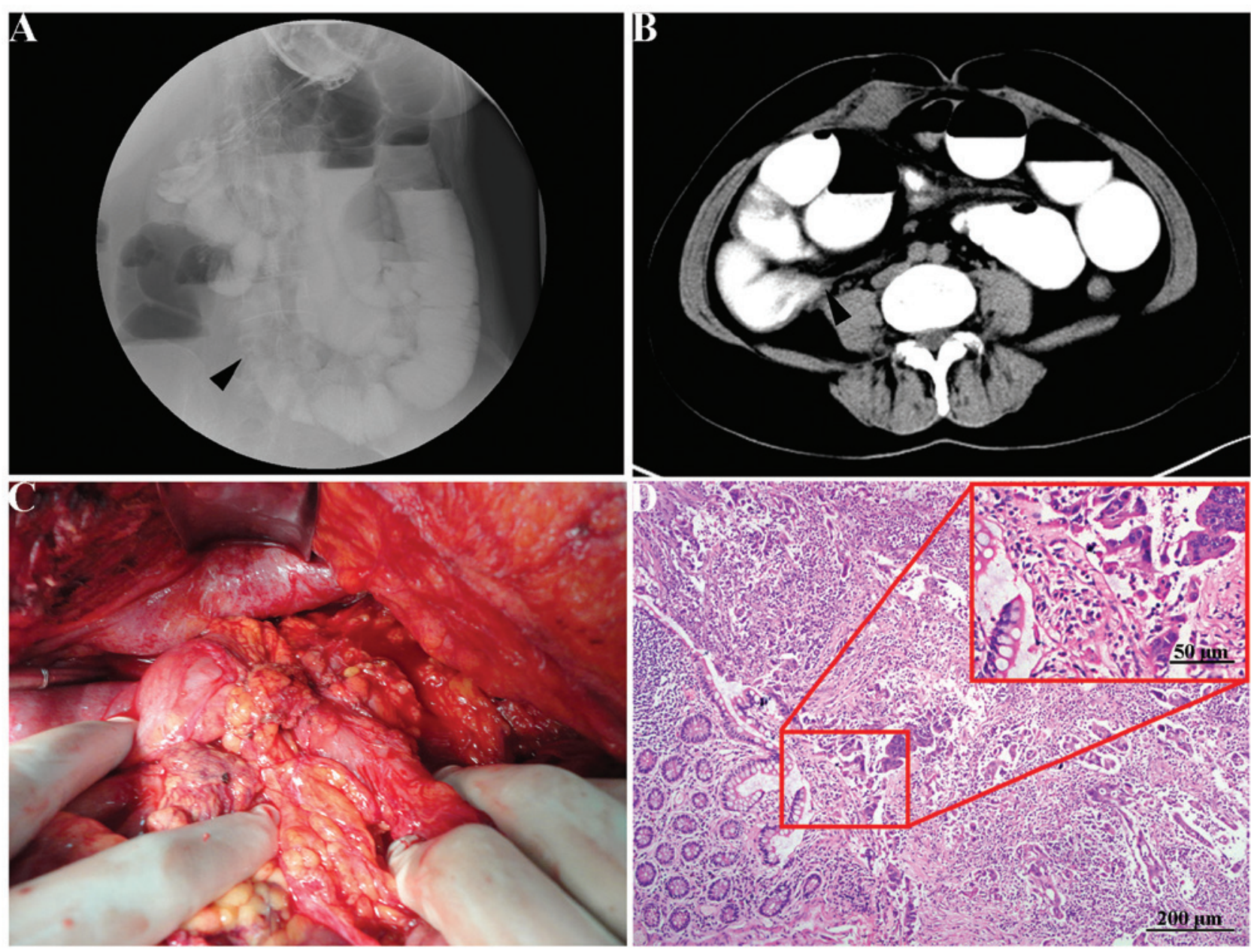

Figure 4. Oral gastrografin radiography (OGR) findings and clinicopathological characteristics in a patient with peritoneal carcinomatosis from mucinous adenocarcinoma of the sigmoid colon. (A) OGR clearly demonstrated limited small intestinal invasion (arrowhead) and the position of the intestinal obstruction, stenosis and peristalsis. (B) Computed tomography revealed the position of ileal obstruction (arrowhead) following OGR. (C) Anatomical position of the ileal obstruction identified during surgery (arrow). (D) The histopathological examination revealed the invasion of the wall of the intestine by mucinous adenocarcinoma (hematoxylin and eosin staining, magnification, x10; scale bar, $200 \mu \mathrm{m}$; inset: magnification, x20).

\section{Discussion}

The present study has developed a simple but useful technique for evaluating the functional impacts of PC on the gastrointestinal system, with the aim to optimize the selection of candidate patients for CRS+HIPEC therapy. Dynamic OGR exhibited several major abnormal radiological findings, including reduced peristalsis/stagnation of the stomach and intestines, gastric wall recess, small intestinal aggregates, luminal stenosis, mucosal disruption and various degrees of mesenteric contracture. These signs are not evenly distributed and are usually more prominent in PC from gastric cancer. Patients with gastric PC exhibited a significantly higher frequency of stomach filling defects $(38.9 \%, 21 / 54)$ and small intestinal aggregates secondary to mesenteric contracture $(70.4 \%, 38 / 54)$ compared with those with PC from other malignancies. This may reflect the different and more aggressive biological behavior of gastric PC, as advanced gastric cancer may result in early intraperitoneal dissemination and trigger mesenteric contracture of the small intestine (17).

There are other non-invasive imaging techniques to display the anatomical or functional abnormalities in the abdomen caused by PC $(18,19)$. Among these, CT-, magnetic resonance imaging (MRI)- and positron emission tomography (PET)/CT-based techniques are commonly used $(20,21)$. CT is the most commonly used technology in clinical practice due to its advantages, such as superior spatial resolution, shorter imaging times (22), wide availability and clinical familiarity (18); its disadvantages, however, are low sensitivity for small tumor nodules and mesenteric deposits. Similarly, MRI has several technical advantages, including superior contrast resolution, multiple imaging types and manipulation of signal intensities; however, its disadvantages include low sensitivity for small tumors, longer imaging times, movement artifacts and lower spatial resolution $(23,24)$. As a functional imaging examination, PET/CT exhibits higher sensitivity compared with MRI and CT, but is associated with technical disadvantages, such as low specificity and spatial resolution for small tumors, high cost and limited availability $(25,26)$. These technologies share one common disadvantage, i.e., they are static technologies showing the sites and severity of PC, but not the dynamic impacts of PC on gastrointestinal function, as respiratory movements may produce artifacts, compromising the image quality. 
In comparison, OGR may help evaluate PC from another perspective. This simple technique may be used to observe the motility of the stomach and intestines, location, mucosal manifestations and extent of tumor invasion under dynamic conditions. Therefore, the OGR-based functional evaluation and the CT- or MRI-based anatomical evaluation may complement each other, making patient selection more reliable.

The practical advantages of OGR are evident. First, the procedure is safe. Barium sulfate is currently a routinely used clinical gastrointestinal contrast agent. This agent is not dissolved in water and must be eliminated from the body. In PC patients, barium sulfate may exacerbate smallintestinal obstruction. As this study focuses on patients with PC or suspected PC with potential intestinal obstruction, barium sulfate should not be used as a contrast agent. Instead, the water-soluble contrast medium gastrografin is a safe, practical and useful alternative. Second, gastrografin is easy to use. The patients undergo a simple preparation, drink the medium and stand before the widely available digital radiography machine for real-time observation of the dynamic functions of the gastrointestinal tract. Other complex procedures may be avoided. Third, the procedure is cost-effective. As PC patients often have to undergo a series of complex examinations and tests before a rational treatment decision can be made, developing a low-cost and easy-to-use radiographic technology meets the clinical priority. Fourth, gastrografin exerts a potential therapeutic effect. Gastrografin is a conventional hyperosmolar contrast medium with pharmacological effects when administered orally. The osmotic pressure is $\sim 1,900 \mathrm{mOsm} / 1$ in the intestinal lumen (6 times more than the extracellular fluid osmotic pressure) (27). Gastrografin may cause drainage of the extracellular and intravascular fluid to the intestine, diluting and increasing the intestinal contents, while reducing local edema, increasing intestinal pressure gradients, stimulating bowel peristalsis and relieving bowel obstruction (28-30). Other effects include inhibition of cholinesterase and release of histamine (31-33) to improve bowel movement. In our experience, a proportion of the patients experienced partial relief of their intestinal obstruction following OGR. These advantages make ORG a practical and valuable technology in the clinical setting.

In conclusion, OGR provides dynamic information on the functional impact of PC on the gastrointestinal system. To the best of our knowledge, this is the first study to assess the functional impacts of PC using a simple technique. However, further studies are required to develop and validate this technology.

\section{Acknowledgements}

This study was supported by the Specialized Research Fund of the Doctoral Program of Higher Education, Ministry of Education of China (grant no. 20120141110042), the Science Fund for Creative Research Groups of the Hubei Province Natural Science Foundation of China (grant no. 2012FFB04314) and the Key Project of the Fundamental Research Fund for the Central Universities (grant no. 303274028).

\section{References}

1. Koppe MJ, Boerman OC, Oyen WJ and Bleichrodt RP: Peritoneal carcinomatosis of colorectal origin: incidence and current treatment strategies. Ann Surg 243: 212-222, 2006.
2. Huang B, Sun Z, Wang Z, et al: Factors associated with peritoneal metastasis in non-serosa-invasive gastric cancer: a retrospective study of a prospectively-collected database. BMC Cancer 13: 57, 2013.

3. Yang XJ, Li Y and Yonemura Y: Cytoreductive surgery plus hyperthermic intraperitoneal chemotherapy to treat gastric cancer with ascites and/or peritoneal carcinomatosis: Results from a Chinese center. J Surg Oncol 101: 457-464, 2010.

4. Yang XJ, Li Y, al-shammaa Hassan AH, et al: Cytoreductive surgery plus hyperthermic intraperitoneal chemotherapy improves survival in selected patients with peritoneal carcinomatosis from abdominal and pelvic malignancies: results of 21 cases. Ann Surg Oncol 16: 345-351, 2009.

5. Huang CQ, Feng JP, Yang XJ and Li Y: Cytoreductive surgery plus hyperthermic intraperitoneal chemotherapy improves survival of patients with peritoneal carcinomatosis from colorectal cancer: a case-control study from a Chinese center. J Surg Oncol 109: 730-739, 2014.

6. Yang XJ, Huang CQ, Suo T, et al: Cytoreductive surgery and hyperthermic intraperitoneal chemotherapy improves survival of patients with peritoneal carcinomatosis from gastric cancer: final results of a phase III randomized clinical trial. Ann Surg Oncol 18: 1575-1581, 2011.

7. Esquivel J, Elias D, Baratti D, Kusamura S and Deraco M: Consensus statement on the loco regional treatment of colorectal cancer with peritoneal dissemination. J Surg Oncol 98: 263-267, 2008.

8. Huang JY, Xu YY, Sun Z, et al: Comparison different methods of intraoperative and intraperitoneal chemotherapy for patients with gastric cancer: a meta-analysis. Asian Pac J Cancer Prev 13: 4379-4385, 2012.

9. Pannu HK, Horton KM and Fishman EK: Thin section dualphase multidetector-row computed tomography detection of peritoneal metastases in gynecologic cancers. J Comput Assist Tomogr 27: 333-340, 2003.

10. Marin D, Catalano C, Baski M, et al: 64-Section multi-detector row $\mathrm{CT}$ in the preoperative diagnosis of peritoneal carcinomatosis: correlation with histopathological findings. Abdom Imaging 35: 694-700, 2010.

11. Franiel T, Diederichs G, Engelken F, Elgeti T, Rost J and Rogalla P: Multi-detector CT in peritoneal carcinomatosis: diagnostic role of thin slices and multiplanar reconstructions. Abdom Imaging 34: 49-54, 2009.

12. de Bree E, Koops W, Kroger R, van Ruth S, Witkamp AJ and Zoetmulder FA: Peritoneal carcinomatosis from colorectal or appendiceal origin: correlation of preoperative CT with intraoperative findings and evaluation of interobserver agreement. J Surg Oncol 86: 64-73, 2004.

13. Coakley FV, Choi PH, Gougoutas CA, et al: Peritoneal metastases: detection with spiral CT in patients with ovarian cancer. Radiology 223: 495-499, 2002.

14. Carmignani CP, Sugarbaker TA,Bromley CM and Sugarbaker PH: Intraperitoneal cancer dissemination: mechanisms of the patterns of spread. Cancer Metastasis Rev 22: 465-472, 2003.

15. Kapoor S, Jain G, Sewkani A, Sharma S, Patel K and Varshney S: Prospective evaluation of oral gastrografin in postoperative small bowel obstruction. J Surg Res 131: 256-260, 2006.

16. Sugarbaker PH: Cytoreductive surgery and peri-operative intraperitoneal chemotherapy as a curative approach to pseudomyxoma peritonei syndrome. Eur J Surg Oncol 27: 239-243, 2001.

17. Roviello F, Caruso S, Neri A and Marrelli D: Treatment and prevention of peritoneal carcinomatosis from gastric cancer by cytoreductive surgery and hyperthermic intraperitoneal chemotherapy: overview and rationale. Eur J Surg Oncol 39: 1309-1316, 2013.

18. Yan TD, Morris DL, Shigeki K, Dario B and Marcello D: Preoperative investigations in the management of peritoneal surface malignancy with cytoreductive surgery and perioperative intraperitoneal chemotherapy: Expert consensus statement. J Surg Oncol 98: 224-227, 2008.

19. Liu Y, Endo Y, Fujita T, et al: Cytoreductive surgery under aminolevulinic acid-mediated photodynamic diagnosis plus hyperthermic intraperitoneal chemotherapy in patients with peritoneal carcinomatosis from ovarian cancer and primary peritoneal carcinoma: Results of a phase I trial. Ann Surg Oncol 21: 4256-4262, 2014

20. Klumpp BD, Aschoff P, Schwenzer N, et al: Peritoneal carcinomatosis: comparison of dynamic contrast-enhanced magnetic resonance imaging with surgical and histopathologic findings. Abdom Imaging 37: 834-842, 2012. 
21. Dirisamer A, Schima W, Heinisch M, et al: Detection of histologically proven peritoneal carcinomatosis with fused ${ }^{18}$ F-FDG-PET/MDCT. Eur J Radiol 69: 536-541, 2009.

22. Funicelli L, Travaini LL, Landoni F, Trifiro G, Bonello L and Bellomi M: Peritoneal carcinomatosis from ovarian cancer: the role of $\mathrm{CT}$ and $\left[{ }^{18} \mathrm{~F}\right]$ FDG-PET/CT. Abdom Imaging 35: 701-707, 2010.

23. Bozkurt M, Doganay S, Kantarci M, et al: Comparison of peritoneal tumor imaging using conventional MR imaging and diffusion-weighted MR imaging with different $\mathrm{b}$ values. Eur J Radiol 80: 224-228, 2011.

24. Wu Y,YueX, Shen W, et al: Diagnostic value of diffusion-weighted MR imaging in thyroid disease: application in differentiating benign from malignant disease. BMC Med Imaging 13: 23, 2013.

25. Sanli Y, Turkmen C, Bakir B, et al: Diagnostic value of PET/CT is similar to that of conventional MRI and even better for detecting small peritoneal implants in patients with recurrent ovarian cancer. Nucl Med Commun 33: 509-515, 2012.

26. Kitajima K, Suenaga Y, Ueno Y, et al: Value of fusion of PET and MRI in the detection of intra-pelvic recurrence of gynecological tumor: comparison with ${ }^{18} \mathrm{~F}-\mathrm{FDG}$ contrast-enhanced PET/CT and pelvic MRI. Ann Nucl Med 28: 25-32, 2014.

27. Khasawneh MA, Ugarte ML, Srvantstian B, Dozois EJ, Bannon MP and Zielinski MD: Role of gastrografin challenge in early postoperative small bowel obstruction. J Gastrointest Surg 18: 363-368, 2014.
28. Ha HK, Kim JS, Lee MS, et al: Differentiation of simple and strangulated small-bowel obstructions: usefulness of known CT criteria. Radiology 204: 507-512, 1997.

29. Fevang BT, Fevang J, Stangeland L, Soreide O, Svanes K and Viste A: Complications and death after surgical treatment of small bowel obstruction: A 35-year institutional experience. Ann Surg 231: 529-537, 2000.

30. Rahmani N, Mohammadpour RA, Khoshnood P, Ahmadi A and Assadpour S: Prospective evaluation of oral gastrografin (R) in the management of postoperative adhesive small bowel obstruction. Indian J Surg 75: 195-199, 2013.

31. Stordahl A, Laerum F, Gjolberg T and Enge I: Water-soluble contrast media in radiography of small bowel obstruction. Comparison of ionic and non-ionic contrast media. Acta Radiol 29: 53-56, 1988.

32. Chen SC, Lin FY, Lee PH, Yu SC, Wang SM and Chang KJ: Water-soluble contrast study predicts the need for early surgery in adhesive small bowel obstruction. Br J Surg 85: 1692-1694, 1998.

33. Assalia A, Schein M, Kopelman D, Hirshberg A and Hashmonai M: Therapeutic effect of oral gastrografin in adhesive, partial small-bowel obstruction: a prospective randomized trial. Surgery 115: 433-437, 1994. 